\title{
Molecular mechanisms of cell death in intervertebral disc degeneration (Review)
}

\author{
FAN ZHANG $^{1,2}$, XUELING ZHAO $^{2}$, HONGXING SHEN $^{1}$ and CAIGUO ZHANG ${ }^{3}$ \\ ${ }^{1}$ Department of Orthopedics, Changhai Hospital Affiliated to The Second Military Medical University, Shanghai 200433; \\ ${ }^{2}$ Department of Orthopedics, The First Affiliated Hospital of Kunming Medical University, \\ Kunming, Yunnan 650032, P.R. China; ${ }^{3}$ Department of Biochemistry and Molecular Genetics, \\ University of Colorado School of Medicine, Aurora, CO 80045, USA
}

Received September 21, 2015; Accepted April 18, 2016

DOI: $10.3892 /$ ijmm.2016.2573

\begin{abstract}
Intervertebral discs (IVDs) are complex structures that consist of three parts, namely, nucleus pulposus, annulus fibrosus and cartilage endplates. With aging, IVDs gradually degenerate as a consequence of many factors, such as microenvironment changes and cell death. Human clinical trial and animal model studies have documented that cell death, particularly apoptosis and autophagy, significantly contribute to IVD degeneration. The mechanisms underlying this phenomenon include the activation of apoptotic pathways and the regulation of autophagy in response to nutrient deprivation and multiple stresses. In this review, we briefly summarize recent progress in understanding the function and regulation of apoptosis and autophagy signaling pathways. In particular, we focus on studies that reveal the functional mechanisms of these pathways in IVD degeneration.
\end{abstract}

\section{Contents}

1. Introduction: Overview of cell death and its intracellular signaling pathways

2. Degeneration of IVD and cell death

3. Apoptosis and IVD degeneration

4. Autophagy and IVD degeneration

5. Conclusion and future aspects

Correspondence to: Dr Hongxing Shen, Department of Orthopedics, Changhai Hospital Affiliated to The Second Military Medical University, Shanghai 200433, P.R. China

E-mail:shenhxgk@126.com

Dr Caiguo Zhang, Department of Biochemistry and Molecular Genetics, University of Colorado School of Medicine, Aurora, CO 80045, USA

E-mail: caiguo.zhang@ucdenver.edu

Key words: apoptosis, autophagy, nucleus pulposus, annulus fibrosus, cartilage endplate

\section{Introduction: Overview of cell death and its intracellular signaling pathways}

Cell death is a fundamental biological process that is required for cellular development. On the basis of its morphological features, cell death can be grouped into three main classes, namely, apoptosis, autophagy and necrosis (1). The deregulation of cell death is associated with the etiology, pathogenesis and treatment of many diseases (2-4), particularly degenerative diseases such as cancer, Alzheimer's disease, heart disease and Parkinson's disease (2,5). Over the past few years, increasing evidence has indicated that cell death contributes to degenerative disc disease (6), spinal degenerative disease, and intervertebral disc (IVD) degeneration $(7,8)$. These findings have led to an improved understanding of the etiology of these diseases as well as providing molecular strategies for therapy. Degenerative changes in IVDs due to aging are clinically important as these changes are associated with back pain. Current understanding of the molecular basis of IVD degeneration is principally focused on the regulation of apoptotic and autophagic pathways.

Apoptosis and its signaling pathways. Apoptosis is a process of programmed cell death that eliminates damaged or non-essential cells without causing local inflammation from cell leakage (9). Apoptotic cells exhibit apparent morphological changes, including cell shrinkage and plasma membrane bubbling as well as nuclear condensation and fragmentation (10). Triggering apoptosis requires a group of cysteine proteases known as caspases, which may be activated through intrinsic and extrinsic signaling pathways (11).

The intrinsic pathway, also known as the mitochondrial pathway, is initiated in the mitochondria (11). As shown in Fig. 1, apoptotic signals, such as DNA damage and cytokine deprivation, activate $\mathrm{p} 53$, which further initiates the intrinsic pathway by upregulating the p53 upregulated modulator of apoptosis (Puma) and Noxa [also known as phorbol-12-myristate-13-acetate-induced protein 1 (PMAIP1)] (12). These two proteins in turn activate pro-apoptotic proteins, such as Bax and Bak, which eventually results in the efflux of cytochrome $c$ (13). Cytochrome $c$ further interacts with the cytosolic protein apoptotic protease activating factor-1 (Apaf-1) to 


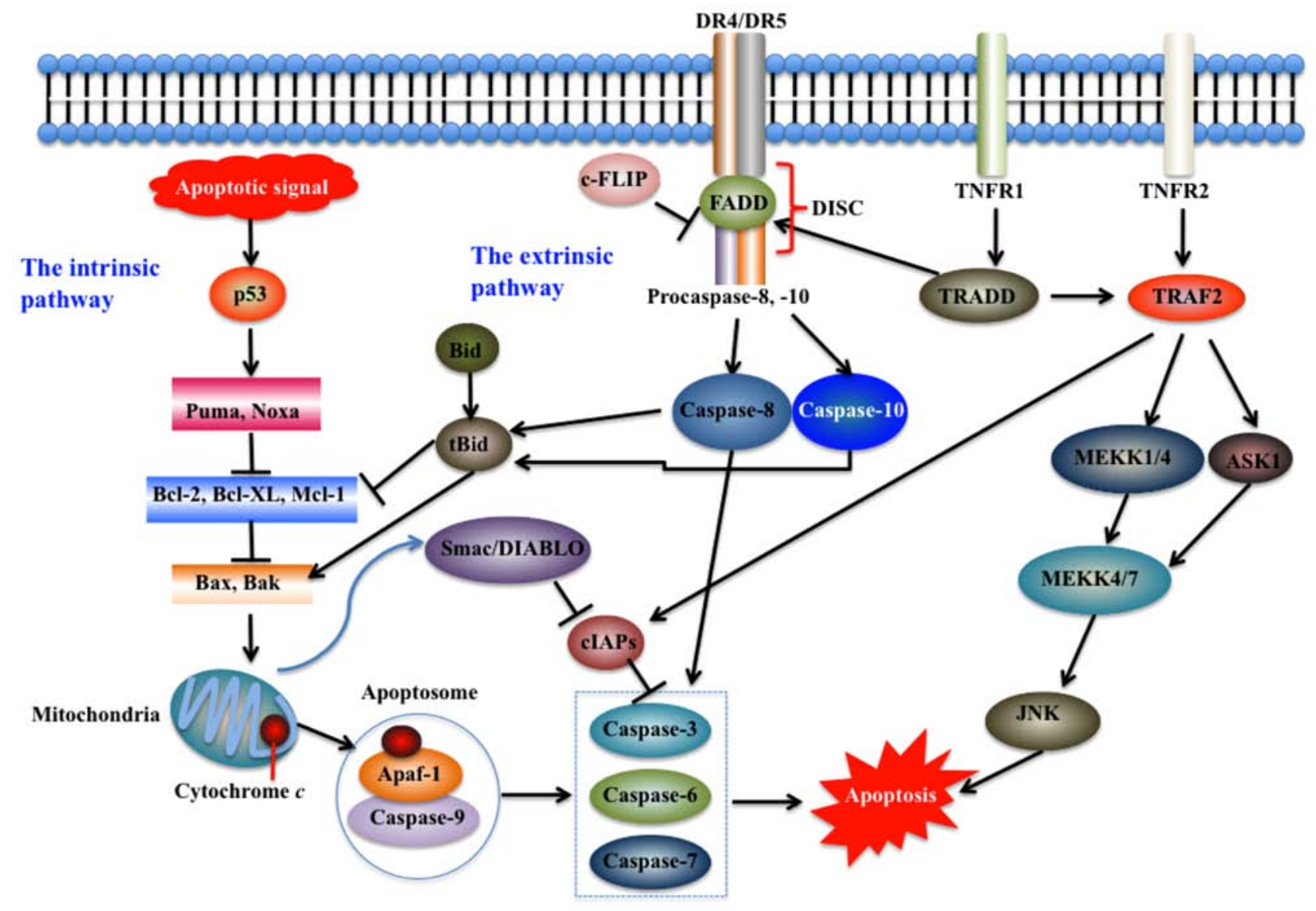

Figure 1. Intrinsic and extrinsic pathways of apoptosis. Apoptosis pathways may be initiated in the mitochondria (intrinsic pathway) and on the plasma membrane by death receptor ligation (extrinsic pathway). The intrinsic pathway is initiated intracellularly, and pro-apoptotic proteins are released from the mitochondria to activate caspase proteases and trigger apoptosis $(12,95)$. The extrinsic pathway mainly comprises two branches, namely, tumor necrosis factor (TNF)-induced apoptosis and Fas-Fas ligand-mediated apoptosis (17). The stimulation of death receptors (DRs) leads to receptor aggregation and recruitment of the adaptor molecule Fas-associated death domain (FADD) and procaspase-8, which subsequently becomes activated and initiates apoptosis by direct cleavage of downstream effector caspases (12). JNK, c-Jun N-terminal kinase; MEKK, mitogen-activated protein kinase kinase; Puma, p53 upregulated modulator of apoptosis; TRADD, TNF receptor-associated death domain protein; TRAF2, TNF receptor-associated factor 2; TNFR1, tumor necrosis factor receptor 1; Apaf-1, apoptotic protease activating factor-1; Bcl-2, B-cell lymphoma-2; Bid, BH3 interacting domain death agonist; Smac/DIABLO, second mitochondria-derived activator of caspase; cIAPs, cellular inhibitor of apoptosis proteins; DISC, death-inducing signaling complex.

recruit caspase-9 to form a complex known as the apoptosome, thereby initiating the activation of the caspase cascade (Fig. 1) (11). Caspase activation leads to nuclear lamin cleavage and nuclear breakdown through caspase- $3,-6$ and $-7(11,13)$. The intrinsic pathway is regulated by various proteins, including nuclear factor $\kappa$-light-chain-enhancer of activated $\mathrm{B}$ cells (NF- $\mathrm{KB}$ ), and $\mathrm{B}-$ cell lymphoma-2 (Bcl-2) protein families. The latter is a large protein family that contains pro-apoptotic members [Bax, Bak, Bad, Bcl-xS, BH3 interacting domain death agonist (Bid), Bik and Bim] and anti-apoptotic members (Hrk, Bcl-2, Bcl-xL, Bcl-W, Bfl-1 and Mcl-1) (13-16). The anti-apoptotic Bcl-2 members repress apoptosis by blocking the release of cytochrome $c$ whereas the pro-apoptotic members promote apoptosis (15). For example, the cytosolic pro-apoptotic protein Bid is cleaved to form a truncated tBid, which further translocates to mitochondria and oligomerizes Bak to release cytochrome $c$ (12). In addition, the mitochondrial protein, second mitochondria-derived activator of caspase (Smac/DIABLO) augments apoptosis by binding to cellular inhibitor of apoptosis proteins (cIAPs) and reversing their grip on several caspases including caspase-3, -6 and -7 (12).
The extrinsic pathway, also known as the cytoplasmic pathway, is initiated by activating pro-apoptotic receptors such as tumor necrosis factor receptor 1 (TNFR1), death receptors (DRs) and Fas on the cell surface (17). This pathway consists of several other proteins, including membrane-bound Fas ligand (FasL), Fas complexes, Fas-associated death domain (FADD), caspase- 8 and -10; these proteins ultimately activate downstream caspases and trigger apoptosis (Fig. 1) $(15,17)$. Previous studies have demonstrated that ligand binding induces receptor clustering and recruitment of the adaptor protein FADD and the initiator caspases- 8 and -10 as procaspases, forming a death-inducing signaling complex (DISC) (12). This event triggers the activation of the apical caspases including caspase- 8 and -10 , driving their autocatalytic processing and release into the cytoplasm, where they activate the effector caspases $-3,-6$ and $-7(18,19)$ (Fig. 1). Several pathways and proteins, such as NF- $\kappa B$, Fas-associated phosphatase-1 (FAP-1), Fas-associated death domain-like interleukin (IL)-1-converting enzyme-like inhibitory protein (FLIP), and decoy receptors (DcR)1 (also known as TRAIL R-3), DcR2 (also known as TRAIL R-4), and DcR3 $(20,21)$, regulate the activation of the extrinsic pathway. 
Although the extrinsic and intrinsic pathways may function separately, crosstalk between these two pathways has been extensively reported. For example, the activation of the extrinsic pathway promotes caspase-8-mediated processing of tBid, which subsequently stimulates Bax and Bak to engage the intrinsic pathway (Fig. 1) (12). Another well-studied crosstalk mechanism between these two pathways regards the stimulation of the intrinsic pathway by the tumor suppressor p53, which also upregulates some of the pro-apoptotic receptors such as DR5 and augments extrinsic signaling (12). In addition, a waxy lipid molecule known as ceramide may directly interfere with the mitochondrion and trigger the activation of the mitochondrial permeability transition (MPT) pore, which further leads to the permeabilization of the mitochondrial outer membrane, the release of mitochondrial intermembrane pro-apoptotic messengers and the induction of apoptotic cascades (22).

Autophagy and signaling pathways. Autophagy involves the degradation of unnecessary or dysfunctional cellular components within lysosomes, and three different forms have been described, namely, macroautophagy, microautophagy and chaperone-mediated autophagy $(23,24)$. Autophagy consists of several critical steps: i) the initiation of autophagy signaling through the unc-51 like autophagy activating kinase 1 complex (24); ii) the regulation of phagophore formation by beclin 1/VPS34 in membranes in response to stress signaling pathways (25); iii) autophagy-related gene (Atg)5-Atg12 conjugation, interaction with $\operatorname{Atg} 16 \mathrm{~L}$, and multimerization at the phagophore $(24,25)$; iv) microtubule-associated protein $1 \mathrm{~A} / 1 \mathrm{~B}$ light chain 3 (LC3) processing and insertion into the extending phagophore membrane (26); v) the degradation of targets and completion of the autophagosome; and vi) the fusion of the autophagosome with lysosomes and proteolytic degradation by lysosomal proteases $(24,27)$.

The regulation of autophagy is complicated and may involve multiple pathways, such as nutrient deprivation, and various stresses (23). Nutrient deprivation may significantly induce autophagosome formation (28). Two well-characterized signaling cascades, including the target of rapamycin (TOR) and Ras-cAMP-dependent protein kinase A (PKA) pathways, sense nutrient status (Fig. 2) (23). TOR regulates nutrient sensing, cell growth, and autophagy (24). TOR activates downstream proteins, including Akt kinase (also known as protein kinase B), phosphoinositide-3 kinase (PI3K) and growth factor receptor (29). Collectively, the Ras/cAMP-dependent PKA signaling pathway plays an important role in glucose sensing in yeast cells and mammals. Under nutrient-rich conditions, two Ras homologs, namely, Ras1 and Ras2, are active and enhance cAMP generation through adenylyl cyclase in yeast (30). Elevated cAMP binds to bypass of cyclic-AMP requirement 1 (Bcyl) and inhibits PKA (23). The constitutive activation of the Ras/PKA pathway may suppress autophagy that is induced by TOR inhibition (23). Various extra- and intracellular stresses, such as endoplasmic reticulum (ER) stress, hypoxia and oxidative stress, potentially induce autophagy (23). ER stress stimulates autophagy through the double stranded RNA-activated protein kinase-like ER kinase-eukaryotic initiation factor-2 $\alpha$ (PERK-eIF2 $\alpha$ ) pathway, the inositol requiring enzyme 1 (IRE1)/c-Jun $\mathrm{N}$-terminal protein kinase $(\mathrm{JNK}) 1$ pathway, and $\mathrm{Ca}^{2+}$ release $(23,31)$ (Fig. 2). The activation of eIF2 $\alpha$ by PERK may enhance the transcription of autophagic genes, such as ATG12 (32). Hypoxia activates autophagy through effects that are dependent on both target genes induced by hypoxia-inducible factor (HIF) and through HIF-independent effects that are mediated by downstream TOR inhibition of AMP-activated protein kinase (AMPK), regulated in development and DNA damage responses 1 (REDD1) and tuberous sclerosis proteins 1 and 2 (TSC1/TSC2) (Fig. 2) (24,28,33). Specific targets of HIF in autophagy include BCL2/adenovirus E1B $19 \mathrm{kDa}$ protein-interacting protein 3 (BNIP3) and BNIP3-like protein (BNIP3L), which are noncanonical members of the Bcl-2 superfamily (Fig. 2) (25,28).

\section{Degeneration of IVDs and cell death}

Structure of IVDs. The IVD is a flexible joint that is localized between adjacent spinal vertebrae $(7,8)$. IVDs consist of the outer endplates, the inner annulus fibrosus (AF), and the central nucleus pulposus (NP) (34). It has been suggested that the endplates absorb the small molecules and nutrients required for the disc cells (35). The AF is the tough, circular exterior of the IVD that surrounds the soft inner NP, and the AF may prevent the NP from herniating or leaking out of the disc by hydraulically sealing the nucleus and by evenly distributing any pressure and force imposed on the IVD (36).

IVD degeneration and signaling pathway regulation. As the human body ages, IVDs gradually degenerate, which leads to degenerative disc disease in some individuals. Various changes in the cellular phenotype and biochemical factors occur during IVD degeneration. These changes mainly include inflammation, matrix degradation, loss of proteoglycan in the NP, disorganization of the concentric lamellae in the AF, spinal instability, disc height loss and prolapse $(37,38)$. Changes to the immune balance of the microenvironment of the disc may cause immune cell infiltration and attack of the NP cells $(39,40)$.

Various intracellular signaling pathways involved in the adaptation of IVD cells to the IVD-specific niche, pain mediators and IVD degeneration have been extensively studied (40). $\mathrm{NF}-\kappa \mathrm{B}$ and mitogen-activated protein kinase (MAPK) pathways regulate proinflammatory mediators such as TNF- $\alpha$, IL- $1 \beta$ and IL-6 (41). The suppression of the NF- $\mathrm{B}$ and MAPK pathways controls anti-inflammatory and anticatabolic conditions during the treatment of IVD herniation and its associated pain (41). MAPK activity also participates in osmoregulation, matrix production, integrin expression and NP cell survival under HIF-1 regulation (42). These findings suggest that $\beta$-catenin is a fundamental factor required to maintain IVD structure and function (43). The Wnt pathway is also suggested to mediate the development and progression of disc diseases (43). $\mathrm{LiCl}$, an activator of the Wnt pathway, accelerated cellular senescence in NP cells $(44,45)$. However, $\beta$-catenin mRNA and protein levels were decreased in NP cells following stimulation with the PKC activator phorbol 12-myristate 13-acetate (PMA) (46). The Notch pathway is also involved in IVD degeneration mediated by proinflammatory cytokines (47). Notch signaling is activated by hypoxia in IVD cells, and the Notch-signaling inhibitor L685458 blocks the activity of Notch-responsive luciferase 


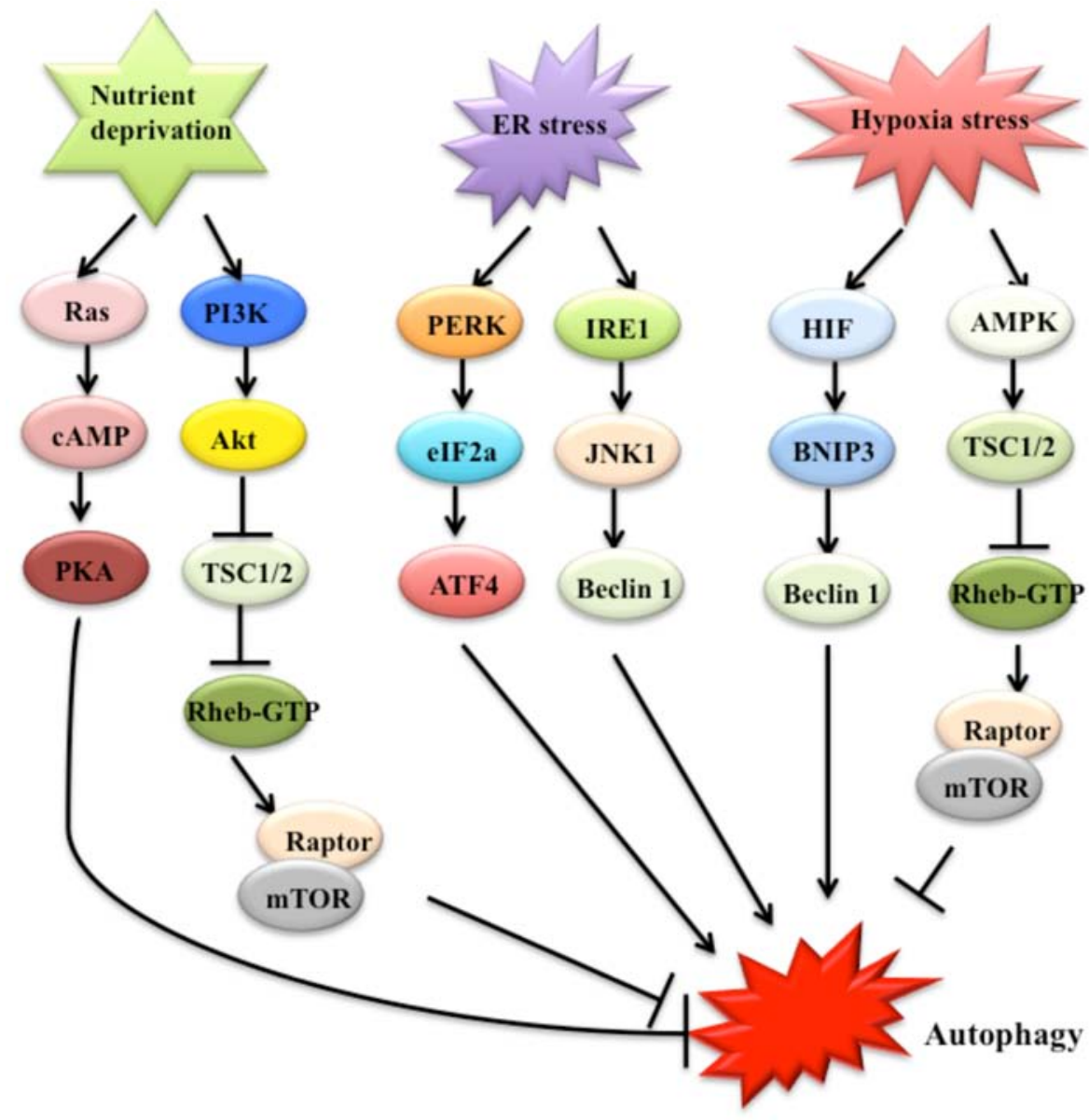

Figure 2. Main signaling pathways involved in autophagy regulation. Multiple factors, including nutrient deprivation, endoplasmic reticulum (ER) stress and hypoxic stress, are involved in autophagy through the mechanisms shown (96). Nutrient deprivation mediates autophagy through the target of rapamycin (TOR) and Ras-cAMP-dependent protein kinase A (PKA) pathways. ER stress stimulates autophagy through the protein kinase-like ER kinase-eukaryotic initiation factor-2 $\alpha$ (PERK-eIF2 $\alpha$ ) and inositol requiring enzyme 1 (IRE1)/c-Jun N-terminal protein kinase 1 (JNK1) pathways. Autophagy is also induced by hypoxia that signals via AMP-activated protein kinase (AMPK) to inhibit mTOR activity or disrupt Bcl-2-beclin-1 interaction and activate beclin-1. PI3K, phosphoinositide 3-kinase; ATF4, activating transcription factor 4; Rheb, Ras homolog enriched in brain; HIF, hypoxia-inducible factor; TSC1/2, tuberous sclerosis proteins 1 and 2; BNIP3, BCL2/adenovirus E1B $19 \mathrm{kDa}$ protein-interacting protein 3.

reporters and reduces the proliferation of AF cells (48). NP cells treated with TNF- $\alpha$ or IL-1 $\beta$ have increased levels of Notch receptors including Notch-1 and -2, the Notch ligand JAGGED2, and target genes such as HES1, HEY1 and HEY2 (49).

Cell death and its causes in IVD degeneration. Apoptosis and autophagy have been commonly observed in degenerative IVDs in clinical trials, animal models and cell culture studies. Cell death may be caused by numerous causes, such as nutrient depletion, biotic and abiotic stress as well as viral infection (49). The cells located at the center of the IVD only acquire nutrients through fluid flow or diffusion through the vertebral endplates and the AF (50). Consequently, nutrients and oxygen tension within the disc are significantly reduced as a result of the long distance from the vasculature to the center of the NP (50). Therefore, the metabolism in disc cells is partly anaerobic, which leads to high lactic acid concentrations and low $\mathrm{pH}$ conditions (50). With disc degeneration, the increased loss of NP proteoglycans reduces the hydrodynamic transfer of axial stress to the outer AF (50). Concurrently, the integrity of the AF is affected by radial fissures. Endplates undergo ossification, which further reduces the nutritional supply to the disc (51). Consequently, changes to the microenvironment, nutrient depletion and stress result in cell death during IVD degeneration.

\section{Apoptosis and IVD degeneration}

Apoptosis has been demonstrated to participate in IVD degeneration for many years. Apoptosis was initially identified using the terminal deoxynucleotidyl transferase-mediated dUTP nick-end labeling (TUNEL) assay; the IVDs from patients were found to have considerably more TUNEL positive cells compared with the healthy control discs (52). Thereafter, numerous studies determined that NP and AF cells undergo apoptosis in degenerative discs through complicated mechanisms.

Apoptosis in NP cells. The excessive apoptosis of NP cells, which produce cartilage-specific extracellular matrix (ECM) components, is an evident cellular and biochemical change which occurs during IVD degeneration $(53,54)$. The dynamic balance between ECM synthesis and degradation is disrupted during IVD degeneration, which results in a gradual loss of disc ECM, structural failure and biomechanical changes (54). 
Both intrinsic and extrinsic pathways of apoptosis play critical roles in NP cell degeneration.

Intrinsic pathway and the degeneration of NP cells. Emerging evidence suggests that the intrinsic pathway of apoptosis participates in NP cell degeneration mainly by regulating the levels of Bcl-2, caspase-3, collagen and aggrecan. Bcl-2 inhibits the intrinsic pathway of apoptosis in various cell systems, including factor-dependent lymphohematopoietic and neural cells (55). Bcl-2 regulates apoptosis by controlling mitochondrial membrane permeability and inhibiting caspase activity either by preventing the release of cytochrome $c$ from the mitochondria and/or by binding to Apaf-1 (55). Notably, Bcl-2 significantly prevents apoptosis during IVD degeneration mainly by inhibiting caspase-3 activity (Fig. 3). It has been demonstrated that NP cells overexpressing $\mathrm{Bcl}$-2 under conditions of serum starvation exhibit reduced apoptosis, decreased mRNA levels of caspase-3 and increased mRNA levels of type II collagen and aggrecan (56). Bcl-2 has been found to bind to nucleotide-binding domain and leucine-rich repeat containing protein 1 (NLRP1) and suppress its activation, thereby inhibiting the release of IL-1 $\beta$, a pro-inflammatory cytokine that is processed to its active form by caspase-1 (57).

Numerous studies have shown that oxidative stress leads to the apoptosis of NP cells during IVD degeneration. NP cells treated with IL-1 $\beta$ exhibited elevated production of nitric oxide (NO) and decreased levels of proteoglycan, which triggered apoptosis (54); apoptosis is increased in NP cells treated with $\mathrm{H}_{2} \mathrm{O}_{2}$ and the mRNA levels of aggrecan and type II collagen are decreased (58). Notably, the deleterious effects of either $\mathrm{H}_{2} \mathrm{O}_{2}$ or IL-1 $\beta$ may be efficiently prevented by glutathione (58), a powerful antioxidant that protects NP cells from apoptosis. Pyrroloquinoline quinone (PQQ), a redox cofactor for bacterial dehydrogenases, potentially scavenges reactive oxygen species (ROS) and inhibits apoptosis (59). PQQ protects rat NP cells against $\mathrm{H}_{2} \mathrm{O}_{2}$-induced apoptosis by inhibiting the intrinsic pathway (59). In the presence of PQQ, ECM production is maintained despite being in an apoptotic environment (59). In addition, the pre-treatment of cells with PQQ increases $B c l-2$ expression, inhibits cytochrome $c$ release, and decreases Bax expression and caspase-3 cleavage (59). These results indicate that glutathione and PQQ are possible therapeutic options for the management of disc degeneration.

Sirtuin-1 (SIRT1), an NAD $\left(^{+}\right)$-dependent deacetylase, has been suggested to reduce apoptosis in NP cells by enhancing the expression of many cartilage-specific ECM genes, such as type II collagen (COL2A1) and aggrecan $(54,60)$. Recent studies have also shown that SIRT1 protects human NP cells from apoptosis by activating the Akt anti-apoptotic signaling pathway (54). In addition, degenerative NP cells obtained from patients have decreased numbers of autophagosomes and low LC3 and beclin 1 levels (6). These findings suggest that autophagy plays an important role in IVD degeneration and that SIRT1 protects the degenerative NP cells in humans against apoptosis by promoting autophagy.

Extrinsic pathway and the degeneration of NP cells. NP cells undergo apoptosis through the extrinsic pathway during IVD degeneration by regulating the levels of Fas and FasL, thereby affecting caspase activities. The expression levels of FasL and Fas were elevated in a co-culture system of human NP cells and human microvascular endothelial (HMEC-1) cells (61). FasL expression in human NP cells prevents angiogenesis in the IVD by inducing Fas-mediated apoptosis with the activation of downstream FADD and caspase-3 (61). The NP is derived from the notochord, a rod-like structure of mesodermal origin (62). Notochordal cells protect NP cells from matrix protein degradation and apoptosis induced by IL-1 $\beta$ and FasL, and this apoptotic process is inhibited by notochordal cell-conditioned medium by suppressing activated caspase- 9 and -3 (63). Bid, cytochrome $c$ and activated caspases-9 and -3 were robustly detected in herniated NP tissues (63). Apoptotic signaling downstream of activated caspase- 9 involves complex interactions between mediators of Smac/DIABLO and X-linked inhibitor of apoptosis protein (XIAP) that control activated caspase-3 signaling (Fig. 3) (64). These findings suggest that Smac/DIABLO and XIAP play a role in the degeneration of NP cells. However, this aspect remains to be clarified. The strong expression of Fas and FasL and the TUNEL-positive staining of a few NP cells in human herniated lumbar IVD tissues indicated the involvement of the DR pathway in IVD degeneration (64). Similar results were also observed in the IVD tissues obtained from patients with scoliosis (65). Recently, a member of a disintegrin and metalloproteinase with thrombospondin motifs (ADAMTS) family, ADAMTS-7, was found to have markedly elevated levels in both human and rat degenerative NP tissues compared with those in normal controls (66). The findings of this study suggest that IL-17A may induce ADAMTS-7 expression through TNF- $\alpha$, which may form a molecular axis in human NP cells (66).

Apoptosis is regulated by miRNAs, which are key post-transcriptional regulators that target the 3'-untranslated regions of the genes that they repress. Aberrant expression profiles of miRNAs are considered as one of the etiologies of IVD degeneration (67). A study examining the miRNA expression profiles revealed that 29 miRNAs are differentially expressed and that miR-155 is significantly downregulated in degenerative NP cells (58). Additional evidence indicated that miR-155 promotes Fas-mediated apoptosis by targeting FADD and caspase-3 (67). Some studies examined the expression of several other miRNAs, such as miR-10b in degenerative NP cells; however, the upstream regulation of miRNAs and their interactions with cytokines remain elusive (67). In addition, miR-27a was recently found to regulate apoptosis in NP cells by targeting PI3K (69). The elevated expression of $m i R-21$ was found in human degenerative NP tissues, and functional analysis revealed that the overexpression of $m i R-21$ increases Akt phosphorylation by targeting phosphatase and tensin homolog (PTEN) (70).

MAPK and the degeneration of NP cells. The MAPK family members are crucial for the maintenance of cell development. Three subfamilies of MAPKs have been identified: extracellular signal-regulated kinases (ERKs), JNKs and p38-MAPKs (71). ERKs are important for cell survival, and JNKs and p38-MAPKs are involved in both the intrinsic and extrinsic pathways of apoptosis (71). The p38-MAPK, JNK1/2 and ERK1/2 signaling pathways exist in NP cells and are required for cell growth, differentiation, and apoptosis (71). A highly osmotic microenvironment may be established during IVD degeneration. Mimicking high-osmolality conditions in vitro activated the p38-MAPK, JNK1/2 and ERK1/2 signaling pathways in rabbit NP cells (72). Furthermore, 


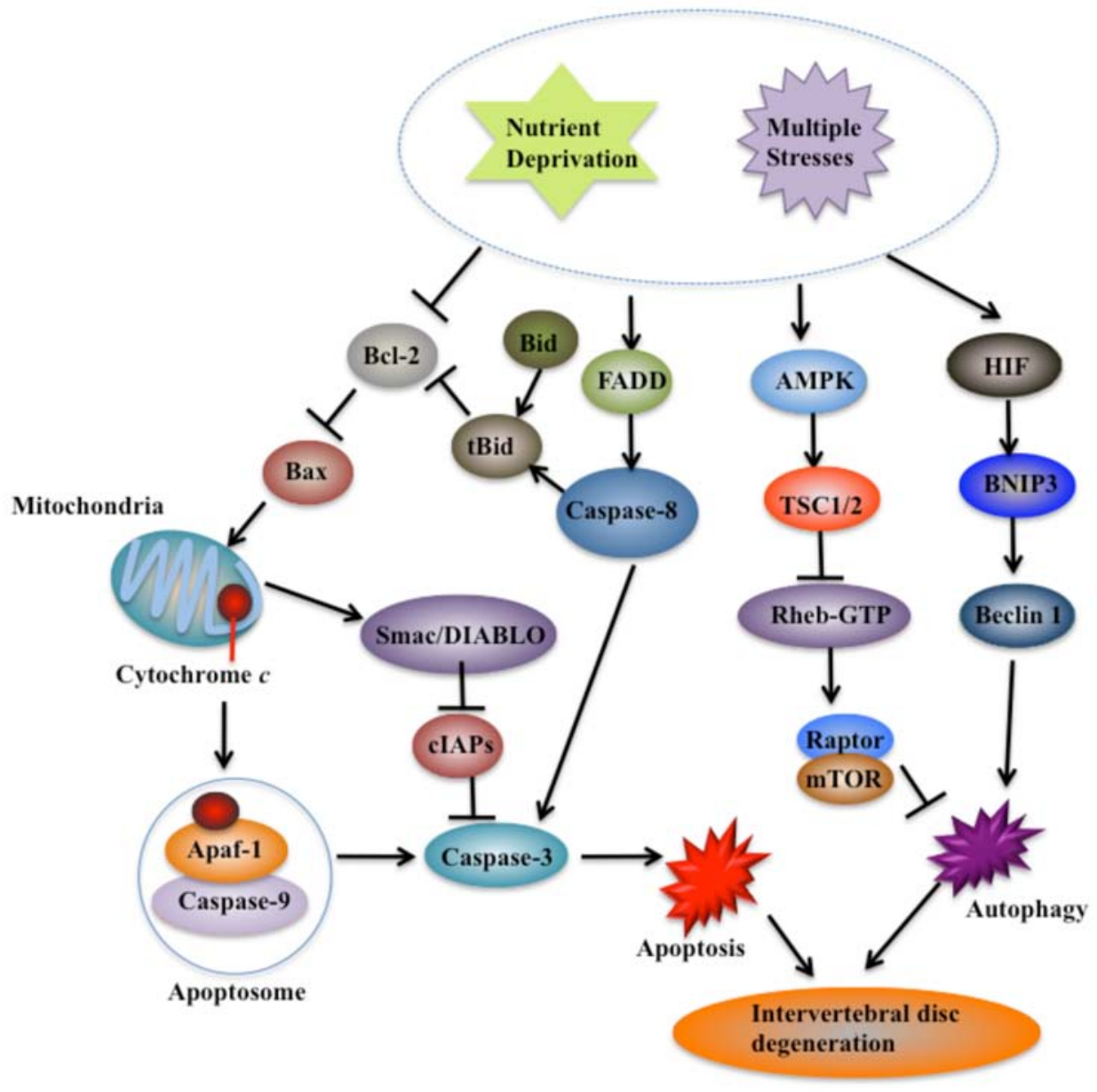

Figure 3. Principal cell death pathways involved in intervertebral disc (IVD) degeneration. With aging, IVDs suffer nutrient deprivation and multiple stresses, and undergo cell death, which eventually results in degeneration. Cells undergo apoptosis through both intrinsic and extrinsic pathways. The leakage of cytochrome $c$ from the mitochondria, the activation of crosstalk between caspase- 8 and BH3 interacting domain death agonist (Bid)-tBid, the important downstream molecules of caspase-9 such as inhibitor of apoptosis protein (IAP), second mitochondria-derived activator of caspase (Smac/DIABLO) and the upstream regulators of mitochondrial maintenance such as Bax and B-cell lymphoma-2 (Bcl-2), play critical roles in IVD degeneration (65,66). Two main autophagic pathways, including signaling via AMP-activated protein kinase (AMPK) to inhibit mTOR activity and the HIF-BINP3-beclin-1 pathway, are also induced during IVD degeneration. BINP3, BCL2/adenovirus E1B $19 \mathrm{kDa}$ protein-interacting protein 3; TSC1/2, tuberous sclerosis proteins 1 and 2; HIF, hypoxia-inducible factor.

activated p38-MAPKs and JNK1/2 may induce cell apoptosis whereas activated ERK1/2 promotes cell survival (72). Recently, $\beta 1$ integrin was found to inhibit apoptosis induced by cyclic stretch in AF cells through the ERK1/2 MAPK pathway, and this process correlates with the activation of caspase-3 (73).

Apoptosis in AF cells. AF cells play an important role in providing the structural properties of the disc, and the apoptosis of AF cells contributes to IVD degeneration through both the intrinsic and extrinsic pathways.

Intrinsic pathway and the degeneration of AF cells. Studies of TUNEL-positive staining in rat AF tissues as well as rabbit models of overload-induced IVD degeneration showed that many cells release anti-cytochrome $c$; however, no anti-FasL-positive cells were identified in these tissues $(74,75)$. These results imply that AF cells undergo apoptosis through the intrinsic pathway under mechanical conditions of overload. In addition, cell proliferation was inhibited after subjecting rabbit AF cells to pressure for 24 or $36 \mathrm{~h}$; this result was associated with increased apoptosis and caspase- 9 activity (75). The activity of caspase-9 is suggested to be proportional to the apoptotic index of rabbit AF cells cultured in silicon elastic membranes; however, no detectable change in caspase- 8 activity was observed in these cells (76). Notably, only caspase-9 inhibitor was capable of suppressing the apoptosis of AF cells induced by cyclic stretch (76). Moreover, it was demonstrated that cyclic stretchinduced apoptosis is partially mediated by ER stress through NO production (77). The cyclic stretch of AF cells caused NO overproduction, the upregulation of ER stress markers (CHOP, GRP78 and caspase-12), mitochondrial depolarization and caspase-9 activation (77). The specific inhibitors of caspase-12 (Z-ATAD-FMK) and caspase-9 (Z-LEHD-FMK) partially suppressed apoptosis (77).

Electroacupuncture (EA) inhibited the apoptosis of AF cells by suppressing the intrinsic pathway in a rat model of IVD degeneration (78). Treatment with EA reduced the number of TUNEL-positive stained cells whereas it increased the number of Bcl-2-positive cells as revealed by immunohistochemical staining (78). Moreover, EA treatment significantly inhibits the activation of caspase- 9 and -3 , and enhances the mRNA and protein levels of Crk and ERK2 (78).

Extrinsic pathway and the degeneration of AF cells. An in vitro study examined the involvement of the extrinsic pathway in IVD degeneration and demonstrated that rabbit 
AF cells undergo increased apoptosis under conditions of serum deprivation (76). This process is associated with the increased activity of caspase- 3 and -8; however, there was no substantial increase in cytochrome $c$ protein levels in the cytosolic fraction (76). The inductive effect of serum deprivation on apoptosis may be reduced by caspase- 8 inhibitor but not by caspase-9 inhibitor (76). Apoptosis in human IVD cells subjected to acute trauma force may be simultaneously and interdependently mediated by extrinsic and intrinsic pathways (79).

Apoptosis in endplate cells. Apoptosis evidently occurs in the cartilaginous endplate cells during IVD degeneration, which results in a marked decrease in cell density (80). The number of TUNEL-positive cells in the cartilaginous endplate increases with age and with the destruction of the cartilaginous endplate following apoptosis (81).

Mechanical stress induced the apoptosis of endplate chondrocytes in organ-cultured mouse IVDs (82). Apoptosis occurred after subjecting the cells to a static mechanical load (82). MAPK inhibitors increase the occurrence of apoptosis, suggesting that MAPKs counteract mechanical stress-induced apoptosis (82). In rat endplate chondrocytes, the increased phosphorylation of JNK, ERK1/2 and p38-MAPK; increased cytochrome $c$ release; and activated caspase- 9 and -3 indicate the occurrence of static mechanical stress-induced apoptosis through the MAPK and intrinsic signaling pathways (82). Treatment with inhibitors of JNK (SP600125), p38-MAPK (SB203580) and ERK (PD98059) prior to mechanical stimulation reversed both the static load-induced apoptosis of chondrocytes and the activation of JNK, p38 MAPK and ERK (82). Collectively, these findings demonstrate that mechanical stress induces apoptosis in rat cervical endplate chondrocytes through the MAPK-mediated mitochondrial apoptotic pathway.

Low levels of fetal bovine serum may induce the apoptosis of rat endplate cells, and serum deprivation leads to the elevated expression of caspase-9, -3 , poly(ADP-ribose) polymerase, cytochrome $c$ and $\operatorname{Bax}$ (83). The caspase-9 inhibitor Z-LEHD-FMK significantly suppressed serum deprivation-induced apoptosis (80). In addition, the activation of acid-sensing ion channel 1a (ASIC1a) in endplate chondrocytes may trigger $\mathrm{Ca}^{2+}$-dependent protease activity and signaling, leading to the apoptosis of endplate chondrocytes in IVDs (84).

\section{Autophagy and IVD degeneration}

Autophagy consists of multiple processes that are highly regulated by Atg proteins and LC3 (23). During autophagy, the cytosolic microtubule-associated protein LC3-I is converted to LC3-II through lipidation, and LC3-II is translocated to the autophagosomal membrane (85). Thus, the conversion of LC3-I to LC3-II and the accumulation of LC3 are widely used as autophagy markers (23). Beclin 1 is a $\mathrm{BH} 3$ member of the Bcl-2 gene family that drives autophagy in mammalian cells (86). Various studies have demonstrated that autophagy occurs in both NP and AF cells. For example, rat NP and AF cells cultured in high glucose concentrations demonstrated the increased expression of beclin 1, LC3 and Atg3, 5, 7 and 12 (85).
Autophagy in NP cells. Rat NP cells exposed to compression undergo ROS-mediated autophagy, which leads to cell degeneration (87). Compression increases the levels of beclin 1 and the processing of LC3B-I to LC3B-II, which is a major step in autophagosome formation (87). The autophagy inhibitor 3-methyladenine (3-MA) attenuates the formation of LC3B and beclin 1 (87). Moreover, glucosamine, an amino sugar and a precursor in the synthesis of glycosylated proteins and lipids, is capable of protecting NP cells and inducing autophagy through the mTORdependent pathway (88). Glucosamine activates autophagy in a dose-dependent manner within $24 \mathrm{~h}$ and inhibits the phosphorylation of mTOR and p70S6K (88). Autophagy in IL-1 $\beta$ - or $\mathrm{H}_{2} \mathrm{O}_{2}$-treated cells is increased by glucosamine (88). Glucosamine attenuates the reduction in aggrecan levels and prevents the apoptosis of NP cells induced by IL-1 $\beta$, whereas 3-MA partially reverses these effects (88). $\mathrm{H}_{2} \mathrm{O}_{2}$ increases the lysosomal membrane permeability in NP cells and subsequently induces apoptosis through the mitochondrial pathway (88). Moreover, $\mathrm{H}_{2} \mathrm{O}_{2}$ stimulates an early autophagic response through the ERK/mTOR signaling pathway (88). The inhibition of autophagy significantly decreases the rate of apoptosis in the cells disrupted with $\mathrm{H}_{2} \mathrm{O}_{2}$ (89). These results suggest that controlling the autophagy response in NP cells under oxidative stress enhances cell survival and probably delays disc degeneration.

Hypoxia facilitates NP cell survival under conditions of serum deprivation by downregulating excessive autophagy through restricting the generation of ROS (90). Appropriate autophagic activity enhances the survival of NP cells under conditions of serum deprivation, whereas excessive autophagy triggers the apoptosis of NP cells (90). Hypoxia facilitates the survival of NP cells in serum deprivation by downregulating excessive autophagy (90). Hypoxia downregulates the autophagic activity of NP cells by restricting the production of ROS and inactivating the AMPK/mTOR signaling pathway, and possibly through a pathway involving HIF-1 $\alpha$ (90). Nutrient starvation may also induce NP cell autophagy by increasing the ratios of LC3-II/LC3-I and beclin-1/ $\beta$-actin, and by producing autophagosomes (90). Treatment with 3-MA may suppress autophagosome formation (90).

Autophagy in AF cells. Under conditions of serum deprivation, autophagy was detected in rat AF cells by transmission electron microscopy (91). IL-1 $\beta$ may dose-dependently enhance the autophagy-induction effect of serum deprivation (91). However, IL-1 $\beta$ alone fails to induce autophagy in AF cells cultured under conditions of serum starvation (91). The suppression of autophagy by 3-MA treatment increases the apoptosis of cells (91). Serum supplementation also partially reverses the incidence of autophagy without affecting the incidence of apoptosis in the same cells. IL-1 $\beta$ dose-dependently upregulates the serum deprivation-induced autophagy of AF cells (91). Autophagy may act as a protective mechanism against apoptosis in AF cells and IVD degeneration.

Autophagy in endplate cells. The IVD obtains nutrients by diffusion from blood vessels through the cartilaginous endplate (92). Thus, endplate calcification may result in disc 
degeneration by decreasing nutrient diffusion and failing to maintain cellular activity and homeostasis, which leads to apoptosis (92). Autophagic activity may correlate with IVD development and degeneration. For example, a previous study demonstrated that autophagy protects the endplate cells from calcification induced by intermittent cyclic mechanical tension (93), and the expression of the autophagy related-genes LC3 and beclin 1 significantly decreases as endplate chondrocyte activity decreases during aging (94).

\section{Conclusion and future aspects}

Cell death is closely associated with the pathology of IVD degeneration. Different types of cells undergo cell death through different signaling pathways in response to various stimuli (Fig. 3). On the basis of current studies, different IVD cell types undergo apoptosis largely through the caspase-9 pathway which is clearly associated with cytochrome $c$ leakage (Fig. 3). In addition, the activation of crosstalk between caspase- 8 and Bid-tBid, the important downstream molecules of caspase-9 such as the inhibitor of apoptosis protein (IAP) and Smac/DIABLO as well as the upstream regulators of mitochondrial maintenance such as Bax and Bcl-2, also play critical roles in IVD degeneration (Fig. 3). Moreover, emerging evidence also suggests that several autophagic pathways, such as the AMPK/mTOR signaling pathway and the HIF-BINP3-beclin 1 pathway, are also induced by multiple stresses (Fig. 3).

Over the past few years, significant progress has been achieved to enhance our understanding of the molecular mechanisms that involve apoptosis and autophagy in IVD degeneration. However, the underlying mechanisms remain incompletely understood. With regard to the apoptotic pathways, future studies should build on current knowledge in order to identify all the key factors of the intrinsic and extrinsic pathways in the different IVD cells, as well as to establish their crosstalk. With regard to the autophagic pathways, current evidence provides either an indication of the factors involved or incomplete signaling pathways. Thus, it is necessary to exert considerable efforts in order to identify the factors that are specifically involved in autophagy during IVD degeneration. Importantly, greater efforts are necessary in order to develop clinical treatments that potentially retard or prevent IVD degeneration in the future.

\section{Acknowledgements}

We apologize to all authors whose contributions were not cited due to space limitations.

\section{References}

1. Kroemer G, Galluzzi L, Vandenabeele P, Abrams J, Alnemri ES, Baehrecke EH, Blagosklonny MV, El-Deiry WS, Golstein P, Green DR, et al; Nomenclature Committee on Cell Death 2009: Classification of cell death: recommendations of the Nomenclature Committee on Cell Death 2009. Cell Death Differ 16: 3-11, 2009.

2. Gorman AM: Neuronal cell death in neurodegenerative diseases: recurring themes around protein handling. J Cell Mol Med 12: 2263-2280, 2008.

3. Xu J, Wang D and Ma W: Cell death in human health and disease. BioMed Res Int 2014: 243017, 2014.
4. Zhang $\mathrm{C}$ and Zhang F: Iron homeostasis and tumorigenesis: molecular mechanisms and therapeutic opportunities. Protein Cell 6: 88-100, 2015.

5. McIlwain DR, Berger T and Mak TW: Caspase functions in cell death and disease. Cold Spring Harb Perspect Biol 7: 7, 2015.

6. Jiang W, Zhang X, Hao J, Shen J, Fang J, Dong W, Wang D, Zhang X, Shui W, Luo Y, et al: SIRT1 protects against apoptosis by promoting autophagy in degenerative human disc nucleus pulposus cells. Sci Rep 4: 7456, 2014.

7. Ding F, Shao ZW and Xiong LM: Cell death in intervertebral disc degeneration. Apoptosis 18: 777-785, 2013.

8. Zhao CQ, Jiang LS and Dai LY: Programmed cell death in intervertebral disc degeneration. Apoptosis 11: 2079-2088, 2006.

9. Taylor RC, Cullen SP and Martin SJ: Apoptosis: controlled demolition at the cellular level. Nat Rev Mol Cell Biol 9: 231-241, 2008.

10. Ashkenazi A and Salvesen G: Regulated cell death: signaling and mechanisms. Annu Rev Cell Dev Biol 30: 337-356, 2014.

11. Elmore S: Apoptosis: a review of programmed cell death. Toxicol Pathol 35: 495-516, 2007.

12. Ashkenazi A: Directing cancer cells to self-destruct with pro-apoptotic receptor agonists. Nat Rev Drug Discov 7: 1001-1012, 2008.

13. Tait SW and Green DR: Mitochondria and cell death: outer membrane permeabilization and beyond. Nat Rev Mol Cell Biol 11: 621-632, 2010.

14. Gogvadze V, Orrenius S and Zhivotovsky B: Multiple pathways of cytochrome $c$ release from mitochondria in apoptosis. Biochim Biophys Acta 1757: 639-647, 2006.

15. Ghobrial IM, Witzig TE and Adjei AA: Targeting apoptosis pathways in cancer therapy. CA Cancer J Clin 55: 178-194, 2005.

16. Kalimuthu S and Se-Kwon K: Cell survival and apoptosis signaling as therapeutic target for cancer: marine bioactive compounds. Int J Mol Sci 14: 2334-2354, 2013.

17. Stanzer S, Janesch B, Resel M, Augustin T, Samonigg H and Bauernhofer T: The role of activation-induced cell death in the higher onset of spontaneous apoptosis of NK cell subsets in patients with metastatic epithelial cancer. Cell Immunol 261: 99-104, 2010

18. Zhang C and Zhang F: The multifunctions of WD40 proteins in genome integrity and cell cycle progression. J Genomics 3: 40-50, 2015.

19. Graumann K, Hippe D, Gross U and Lüder CG: Mammalian apoptotic signalling pathways: multiple targets of protozoan parasites to activate or deactivate host cell death. Microbes Infect 11: 1079-1087, 2009.

20. Plati J, Bucur O and Khosravi-Far R: Apoptotic cell signaling in cancer progression and therapy. Integr Biol Camb 3: 279-296, 2011.

21. Fulda S and Debatin KM: Extrinsic versus intrinsic apoptosis pathways in anticancer chemotherapy. Oncogene 25: 4798-4811, 2006.

22. Klener P Jr, Andera L, Klener P, Necas E and Zivný J: Cell death signalling pathways in the pathogenesis and therapy of haematologic malignancies: overview of apoptotic pathways. Folia Biol (Praha) 52: 34-44, 2006.

23. Ouyang L, Shi Z, Zhao S, Wang FT, Zhou TT, Liu B and Bao JK: Programmed cell death pathways in cancer: a review of apoptosis, autophagy and programmed necrosis. Cell Prolif 45: 487-498, 2012.

24. He C and Klionsky DJ: Regulation mechanisms and signaling pathways of autophagy. Annu Rev Genet 43: 67-93, 2009.

25. Yamamoto A and YueZ: Autophagy and its normal and pathogenic states in the brain. Annu Rev Neurosci 37: 55-78, 2014.

26. Glick D, Barth S and Macleod KF: Autophagy: cellular and molecular mechanisms. J Pathol 221: 3-12, 2010.

27. Matsushita M, Suzuki NN, Obara K, Fujioka Y, Ohsumi Y and Inagaki F: Structure of Atg5.Atg16, a complex essential for autophagy. J Biol Chem 282: 6763-6772, 2007.

28. Pattingre S, Espert L, Biard-Piechaczyk M and Codogno P: Regulation of macroautophagy by mTOR and Beclin 1 complexes. Biochimie 90: 313-323, 2008.

29. Russell RC, Yuan HX and Guan KL: Autophagy regulation by nutrient signaling. Cell Res 24: 42-57, 2014.

30. Richardson CJ, Schalm SS and Blenis J: PI3-kinase and TOR: PIKTORing cell growth. Semin Cell Dev Biol 15: 147-159, 2004.

31. D'Souza CA and Heitman J: Conserved cAMP signaling cascades regulate fungal development and virulence. FEMS Microbiol Rev 25: 349-364, 2001.

32. Shen S, Zhang Y, Wang Z, Liu R and Gong X: Bufalin induces the interplay between apoptosis and autophagy in glioma cells through endoplasmic reticulum stress. Int J Biol Sci 10: 212-224, 2014. 
33. Zhang C, Liu G and Huang M: Ribonucleotide reductase metallocofactor: Assembly, maintenance and inhibition. Front Biol (Beijing) 9: 104-113, 2014.

34. Le Maitre CL, Binch AL, Thorpe AA and Hughes SP: Degeneration of the intervertebral disc with new approaches for treating low back pain. J Neurosurg Sci 59: 47-61, 2015

35. Bron JL, Helder MN, Meisel HJ, Van Royen BJ and Smit TH: Repair, regenerative and supportive therapies of the annulus fibrosus: achievements and challenges. Eur Spine J 18: 301-313, 2009.

36. Moore RJ: The vertebral endplate: disc degeneration, disc regeneration. Eur Spine J 15 (Suppl 3): S333-S337, 2006.

37. Sivan SS, Hayes AJ, Wachtel E, Caterson B, Merkher Y, Maroudas A, Brown S and Roberts S: Biochemical composition and turnover of the extracellular matrix of the norma and degenerate intervertebral disc. Eur Spine J 23 (Suppl 3): S344-S353, 2014.

38. Iatridis JC, Nicoll SB, Michalek AJ, Walter BA and Gupta MS: Role of biomechanics in intervertebral disc degeneration and regenerative therapies: what needs repairing in the disc and what are promising biomaterials for its repair? Spine J 13: 243-262, 2013.

39. Urban JP and Roberts S: Degeneration of the intervertebral disc. Arthritis Res Ther 5: 120-130, 2003.

40. Liu ZH, Sun Z, Wang HQ, Ge J, Jiang TS, Chen YF, Ma Y, Wang C, Hu S, Samartzis D and Luo ZJ: FasL expression on human nucleus pulposus cells contributes to the immune privilege of intervertebral disc by interacting with immunocytes. Int J Med Sci 10: 1053-1060, 2013

41. Hiyama A, Sakai D and Mochida J: Cell signaling pathways related to pain receptors in the degenerated disk. Global Spine J 3: 165-174, 2013.

42. Risbud MV, Schipani E and Shapiro IM: Hypoxic regulation of nucleus pulposus cell survival: from niche to notch. Am J Pathol 176: 1577-1583, 2010.

43. Kondo N, Yuasa T, Shimono K, Tung W, Okabe T, Yasuhara R, Pacifici $M$, Zhang $Y$, Iwamoto $M$ and Enomoto-Iwamoto $M$ : Intervertebral disc development is regulated by $\mathrm{Wnt} / \beta$-catenin signaling. Spine 36: E513-E518, 2011.

44. Hiyama A, Sakai D, Risbud MV, Tanaka M, Arai F, Abe K and Mochida J: Enhancement of intervertebral disc cell senescence by $\mathrm{WNT} / \beta$-catenin signaling-induced matrix metalloproteinase expression. Arthritis Rheum 62: 3036-3047, 2010.

45. Arai F, Hiyama A, Sakai D, Yokoyama K and Mochida J: The expression and role of non-canonical (PKC) signaling in nucleus pulposus cell metabolism. J Orthop Res 30: 1478-1485, 2012

46. Risbud MV and Shapiro IM: Role of cytokines in intervertebral disc degeneration: pain and disc content. Nat Rev Rheumatol 10: 44-56, 2014

47. Hiyama A, Skubutyte R, Markova D, Anderson DG, Yadla S, Sakai D, Mochida J, Albert TJ, Shapiro IM and Risbud MV: Hypoxia activates the Notch signaling pathway in cells of the intervertebral disc: implications in degenerative disc disease. Arthritis Rheum 63: 1355-1364, 2011.

48. Wang H, Tian Y, Wang J, Phillips KL, Binch AL, Dunn S, Cross A, Chiverton N, Zheng Z, Shapiro IM, et al: Inflammatory cytokines induce NOTCH signaling in nucleus pulposus cells: implications in intervertebral disc degeneration. J Biol Chem 288: 16761-16774, 2013

49. Liu Y and Levine B: Autosis and autophagic cell death: the dark side of autophagy. Cell Death Differ 22: 367-376, 2015.

50. Sakai D and Grad S: Advancing the cellular and molecular therapy for intervertebral disc disease. Adv Drug Deliv Rev 84 $159-171,2015$.

51. Wang HQ and Samartzis D: Clarifying the nomenclature of intervertebral disc degeneration and displacement: from bench to bedside. Int J Clin Exp Pathol 7: 1293-1298, 2014.

52. Gruber HE and Hanley EN Jr: Analysis of aging and degeneration of the human intervertebral disc. Comparison of surgical specimens with normal controls. Spine 23: 751-757, 1998.

53. Gruber HE and Hanley EN Jr: Biologic strategies for the therapy of intervertebral disc degeneration. Expert Opin Biol Ther 3 : 1209-1214, 2003.

54. Wang D, Hu Z, Hao J, He B, Gan Q, Zhong X, Zhang X, Shen J, Fang J and Jiang W: SIRT1 inhibits apoptosis of degenerative human disc nucleus pulposus cells through activation of Akt pathway. Age (Dordr) 35: 1741-1753, 2013.

55. Cory S, Huang DC and Adams JM: The Bcl-2 family: roles in cell survival and oncogenesis. Oncogene 22: 8590-8607, 2003

56. Sudo $\mathrm{H}$ and Minami A: Regulation of apoptosis in nucleus pulposus cells by optimized exogenous Bcl-2 overexpression. J Orthop Res 28: 1608-1613, 2010
57. Gabelloni ML, Sabbione F, Jancic C, Fuxman Bass J, Keitelman I, Iula L, Oleastro M, Geffner JR and Trevani AS: NADPH oxidase derived reactive oxygen species are involved in human neutrophil IL-1 $\beta$ secretion but not in inflammasome activation. Eur J Immunol 43: 3324-3335, 2013.

58. Yang D, Wang D, Shimer A, Shen FH, Li X and Yang X Glutathione protects human nucleus pulposus cells from cell apoptosis and inhibition of matrix synthesis. Connect Tissue Res 55: 132-139, 2014.

59. Yang L, Rong Z, Zeng M, Cao Y, Gong X, Lin L, Chen Y, Cao W, Zhu L and Dong W: Pyrroloquinoline quinone protects nucleus pulposus cells from hydrogen peroxide-induced apoptosis by inhibiting the mitochondria-mediated pathway. Eur Spine J 24 1702-1710, 2015

60. Dvir-Ginzberg M, Gagarina V, Lee EJ and Hall DJ: Regulation of cartilage-specific gene expression in human chondrocytes by SirT1 and nicotinamide phosphoribosyltransferase. J Biol Chem 283: 36300-36310, 2008

61. Sun Z, Wan ZY, Guo YS, Wang HQ and Luo ZJ: FasL on human nucleus pulposus cells prevents angiogenesis in the disc by inducing Fas-mediated apoptosis of vascular endothelial cells. Int J Clin Exp Pathol 6: 2376-2385, 2013.

62. Risbud MV and Shapiro IM: Notochordal cells in the adult intervertebral disc: new perspective on an old question. Crit Rev Eukaryot Gene Expr 21: 29-41, 2011

63. Erwin WM, Islam D, Inman RD, Fehlings MG and Tsui FW: Notochordal cells protect nucleus pulposus cells from degradation and apoptosis: implications for the mechanisms of intervertebral disc degeneration. Arthritis Res Ther 13: R215, 2011.

64. Park JB, Kim KW, Han CW and Chang H: Expression of Fas receptor on disc cells in herniated lumbar disc tissue. Spine 26 142-146, 2001

65. Chen B, Fellenberg J, Wang H, Carstens C and Richter W: Occurrence and regional distribution of apoptosis in scoliotic discs. Spine 30: 519-524, 2005.

66. Wang SS, Zhang W, Zhang YQ, Zhao Y, Liu Y, Li JK, Zhang HX, Cheng L and Nie L: IL-17A enhances ADAMTS-7 expression through regulation of TNF- $\alpha$ in human nucleus pulposus cells J Mol Histol 46: 475-483, 2015.

67. Wang HQ, Yu XD, Liu ZH, Cheng X, Samartzis D, Jia LT, Wu SX, Huang J, Chen J and Luo ZJ: Deregulated miR-155 promotes Fas-mediated apoptosis in human intervertebral disc degeneration by targeting FADD and caspase-3. J Pathol 225: 232-242, 2011.

68. Zhao B, Yu Q, Li H, Guo X and He X: Characterization of microRNA expression profiles in patients with intervertebral disc degeneration. Int J Mol Med 33: 43-50, 2014.

69. Liu G, Cao P, Chen H, Yuan W, Wang J and Tang X: MiR-27a regulates apoptosis in nucleus pulposus cells by targeting PI3K. PLoS One 8: e75251, 2013.

70. Liu H, Huang X, Liu X, Xiao S, Zhang Y, Xiang T, Shen X, Wang G and Sheng B: miR-21 promotes human nucleus pulposus cell proliferation through PTEN/AKT signaling. Int J Mol Sci 15: 4007-4018, 2014

71. Wada T and Penninger JM: Mitogen-activated protein kinases in apoptosis regulation. Oncogene 23: 2838-2849, 2004.

72. Dong ZH, Wang DC, Liu TT, Li FH, Liu RL, Wei JW and Zhou CL: The roles of MAPKs in rabbit nucleus pulposus cell apoptosis induced by high osmolality. Eur Rev Med Pharmacol Sci 18: 2835-2845, 2014

73. Zhang K, Ding W, Sun W, Sun XJ, Xie YZ, Zhao CQ and Zhao J: Betal integrin inhibits apoptosis induced by cyclic stretch in annulus fibrosus cells via ERK1/2 MAPK pathway. Apoptosis: Oct 14, 2015 (Epub ahead of print).

74. Yurube T, Hirata H, Kakutani K, Maeno K, Takada T, Zhang Z, Takayama K, Matsushita T, Kuroda R, Kurosaka M and Nishida K: Notochordal cell disappearance and modes of apoptotic cell death in a rat tail static compression-induced disc degeneration model. Arthritis Res Ther 16: R31, 2014

75. Xie M, Yang S, Win HL, Xiong L, Huang J and Zhou J: Rabbit annulus fibrosus cell apoptosis induced by mechanical overload via a mitochondrial apoptotic pathway. J Huazhong Univ Sci Technolog Med Sci 30: 379-384, 2010.

76. Rannou F, Lee TS, Zhou RH, Chin J, Lotz JC, MayouxBenhamou MA, Barbet JP, Chevrot A and Shyy JY: Intervertebral disc degeneration: the role of the mitochondrial pathway in annulus fibrosus cell apoptosis induced by overload. Am J Pathol 164: 915-924, 2004.

77. Zhang YH, Zhao CQ, Jiang LS and Dai LY: Cyclic stretchinduced apoptosis in rat annulus fibrosus cells is mediated in part by endoplasmic reticulum stress through nitric oxide production. Eur Spine J 20: 1233-1243, 2011. 
78. Liao J, Ke M, Xu T and Lin L: Electroacupuncture inhibits apoptosis in annulus fibrosis cells through suppression of the mitochondria-dependent pathway in a rat model of cervical intervertebral disc degradation. Genet Mol Biol 35: 686-692, 2012.

79. Alkhatib B, Rosenzweig DH, Krock E, Roughley PJ, Beckman L, Steffen T, Weber MH, Ouellet JA and Haglund L: Acute mechanical injury of the human intervertebral disc: link to degeneration and pain. Eur Cell Mater 28: 98-111, 2014.

80. Zhao CQ, Wang LM, Jiang LS and Dai LY: The cell biology of intervertebral disc aging and degeneration. Ageing Res Rev 6 : 247-261, 2007.

81. Ariga K, Miyamoto S, Nakase T, Okuda S, Meng W, Yonenobu K and Yoshikawa $\mathrm{H}$ : The relationship between apoptosis of endplate chondrocytes and aging and degeneration of the intervertebral disc. Spine 26: 2414-2420, 2001.

82. Ariga K, Yonenobu K, Nakase T, Hosono N, Okuda S, Meng W, Tamura Y and Yoshikawa H: Mechanical stress-induced apoptosis of endplate chondrocytes in organ-cultured mouse intervertebral discs: an ex vivo study. Spine 28: 1528-1533, 2003.

83. Li D, Zhu B, Ding L, Lu W, Xu G and Wu J: Role of the mitochondrial pathway in serum deprivation-induced apoptosis of rat endplate cells. Biochem Biophys Res Commun 452: 354-360, 2014.

84. Li X, Wu FR, Xu RS, Hu W, Jiang DL, Ji C, Chen FH and Yuan FL: Acid-sensing ion channel la-mediated calcium influx regulates apoptosis of endplate chondrocytes in intervertebral discs. Expert Opin Ther Targets 18: 1-14, 2014.

85. Kong CG, Park JB, Kim MS and Park EY: High glucose accelerates autophagy in adult rat intervertebral disc cells. Asian Spine J 8: 543-548, 2014

86. Kang R, Zeh HJ, Lotze MT and Tang D: The Beclin 1 network regulates autophagy and apoptosis. Cell Death Differ 18: 571-580, 2011.

87. Ma KG, Shao ZW, Yang SH, Wang J, Wang BC, Xiong LM, Wu Q and Chen SF: Autophagy is activated in compression-induced cell degeneration and is mediated by reactive oxygen species in nucleus pulposus cells exposed to compression. Osteoarthritis Cartilage 21: 2030-2038, 2013.
88. Jiang L, Jin Y, Wang H, Jiang Y and Dong J: Glucosamine protects nucleus pulposus cells and induces autophagy via the mTOR-dependent pathway. J Orthop Res 32: 1532-1542, 2014.

89. Chen JW, Ni BB, Li B, Yang YH, Jiang SD and Jiang LS: The responses of autophagy and apoptosis to oxidative stress in nucleus pulposus cells: implications for disc degeneration. Cell Physiol Biochem 34: 1175-1189, 2014.

90. Chen JW, Ni BB, Zheng XF, Li B, Jiang SD and Jiang LS: Hypoxia facilitates the survival of nucleus pulposus cells in serum deprivation by down-regulating excessive autophagy through restricting ROS generation. Int J Biochem Cell Biol 59: $1-10,2015$.

91. Shen C, Yan J, Jiang LS and Dai LY: Autophagy in rat annulus fibrosus cells: evidence and possible implications. Arthritis Res Ther 13: R132, 2011.

92. Jackson AR, Huang CY and Gu WY: Effect of endplate calcification and mechanical deformation on the distribution of glucose in intervertebral disc: a 3D finite element study. Comput Methods Biomech Biomed Engin 14: 195-204, 2011.

93. Xu HG, Yu YF, Zheng Q, Zhang W, Wang CD, Zhao XY, Tong WX, Wang H, Liu P and Zhang XL: Autophagy protects end plate chondrocytes from intermittent cyclic mechanical tension induced calcification. Bone 66: 232-239, 2014

94. Yu YF, Xu HG, Wang H, Zhang W, Xiong SL and Zhang M: Change of autophagy in endplate chondrocytes of rats during aging process. Zhonghua Yi Xue Za Zhi 93: 3632-3635, 2013 (In Chinese).

95. Fadeel B and Orrenius S: Apoptosis: a basic biological phenomenon with wide-ranging implications in human disease. J Intern Med 258: 479-517, 2005.

96. Xu Y, Xia X and Pan H: Active autophagy in the tumor microenvironment: a novel mechanism for cancer metastasis. Oncol Lett 5: 411-416, 2013. 